HUTP-98/A082

\title{
High-Energy Tests of Lorentz Invariance
}

\author{
Sidney Coleman and Sheldon L. Glashow \\ Lyman Laboratory of Physics \\ Harvard University \\ Cambridge, MA 02138
}

\begin{abstract}
We develop a perturbative framework with which to discuss departures from exact Lorentz invariance and explore their potentially observable ramifications. Tiny noninvariant terms introduced into the standard model Lagrangian are assumed to be renormalizable (dimension $\leq 4$ ), invariant under $S U(3) \otimes S U(2) \otimes U(1)$ gauge transformations, and rotationally and translationally invariant in a preferred frame. There are a total of 46 independent CPT-even perturbations of this kind, all of which preserve anomaly cancellation. They define the energy-momentum eigenstates and their maximal attainable velocities in the high-energy limit. The effects of these perturbations increase rapidly with energy in the preferred frame, more rapidly than those of CPT-odd perturbations. Our analysis of Lorentz-violating kinematics reveals several striking new phenomena that are relevant both to cosmic-ray physics (e.g., by undoing the GZK cutoff) and neutrino physics (e.g., by generating novel types of neutrino oscillations). These may lead to new and sensitive high-energy tests of special relativity.
\end{abstract}

$18 / 1 / 99$ 


\section{Introduction}

Experimental tests of Lorentz invariance have become remarkably accurate. To give a quantitative measure of this accuracy, one imagines adding tiny Lorentz-violating terms to a conventional Lagrangian. Experiments can test Lorentz invariance by setting upper bounds to the coefficients of these terms. One common choice [1] is to alter the coefficient of the square of the magnetic field in the Lagrangian of quantum electrodynamics:

$$
\vec{B}^{2} \rightarrow(1+\epsilon) \vec{B}^{2} .
$$

Among other effects, this term causes the velocity of light $c$, given by $c^{2}=1+\epsilon$, to differ from the maximum attainable velocity of a material body, which remains equal to one. (Shortly we shall consider more general Lorentz-violating perturbations.)

The perturbation (1.1) breaks Lorentz invariance. It is translationally and rotationally invariant in the frame in which we are working ("the preferred frame") but not in any other frame. If the preferred frame is the one in which the cosmic microwave background is isotropic, tiny and calculable anisotropies should appear in laboratory experiments. Highprecision spectroscopic experiments that fail to find such anisotropies [2] [3] set the bound $\left|1-c^{2}\right|=|\epsilon|<6 \times 10^{-22}$.

In a note published last year [4] we pointed out that a better bound could be obtained from a very different sort of experiment if $c<1$. In this case a charged particle traveling faster than light rapidly radiates photons until it is no longer superluminal. Thus no primary cosmic-ray proton can have energy greater than $M / \sqrt{1-c^{2}}=M|\epsilon|^{-1 / 2}$, where $M$ is the proton mass. Because primary protons with energies up to $10^{20} \mathrm{eV}$ are seen, we set the bound $1-c^{2}<10^{-23}$, almost an order of magnitude stronger than the atomicphysics bound. High accuracy is obtained from high energy rather than high precision. Moreover our bound requires no assumption about our velocity in the preferred frame.

This effect, which we call vacuum Čerenkov radiation, is absent below a characteristic energy and turns on abruptly once that energy is reached. Such is not always the case, as the following example shows.

Let $\Psi$ denote a set of $n$ complex scalar fields assembled into a column vector. If we assume invariance under the $U(1)$ group $\Psi \rightarrow e^{-i \lambda} \Psi$, the most general free Lagrangian is:

$$
\mathcal{L}=\partial_{\mu} \Psi^{*} Z \partial^{\mu} \Psi-\Psi^{*} M^{2} \Psi,
$$


where $Z$ and $M^{2}$ are positive Hermitian matrices. We can always linearly transform the fields to make $Z$ the identity and $M^{2}$ diagonal, thus obtaining the standard theory of $n$ decoupled free fields. Consider adding to the Lagrangian the Lorentz-violating term:

$$
\mathcal{L} \rightarrow \mathcal{L}+\partial_{i} \Psi \epsilon \partial^{i} \Psi
$$

where $\epsilon$ is a Hermitian matrix. If $\epsilon$ does not commute with $M^{2}$, there is no way to disentangle the fields. The single-particle energy-momentum eigenstates go from eigenstates of $M^{2}$ at low momenta to eigenstates of $\epsilon$ at high momenta. In contrast to vacuum Čerenkov radiation, this effect, which we call velocity mixing, turns on gradually. Gradual effects allow one to obtain high accuracy by combining moderately high energies with moderately high precision.

A more striking gradual effect appears if this system is minimally coupled to electromagnetism. In this case, a meson can decay to a less massive meson plus a photon at a rate growing with the cube of the energy. Analogous terms in the standard model can drive the otherwise-forbidden decay $\mu^{+} \rightarrow e^{+}+\gamma$ and the $0-0$ transition $K^{+} \rightarrow \pi^{+}+\gamma$.

This example shows that what meant by "high energy" in this context depends very much on the details of the system under consideration. For simplicity, suppose $n=2$ and let $M^{2}=\operatorname{diag}\left(M_{1}^{2}, M_{2}^{2}\right)$. The transition from eigenstates of $M^{2}$ to those of $\epsilon$ occurs at energies $\sim \sqrt{\left(M_{1}^{2}-M_{2}^{2}\right) / \epsilon_{12}}$. For the neutral kaon system, this energy is many orders of magnitude less than the characteristic energy of vacuum Cerenkov radiation (if the dimensionless invariance-violating parameters in the two processes are comparable).

These are just illustrative examples. In $\S 2$ we study all local Lorentz-invariance violating interactions that are rotationally and translationally invariant in a preferred frame and of renormalizable type (i.e., having mass dimension $\leq 4)$. 1 (Some of the results in $\S 2.1$ and $\S 2.2$ were first established by Kostelecký and Colladay [5]. We derive them anew here, both for completeness and because we wish to emphasize features relevant to high-energy tests.)

Lorentz-violating perturbations can be divided into two classes, depending on whether they are even or odd under CPT. For a state with energy $E$, we show in $\S 2.1$ that the

1 The condition of renormalizability can be given the usual justification: If we assume that breaking of Lorentz invariance occurs at some very small distance scale, only the renormalizable interactions survive the renormalization-group running of the couplings to experimentally accessible scales. 
expectation values of the CPT-even interactions grow like $E^{2}$ for large $E$, while those of the CPT-odd interactions grow like $E$. Because we are interested in effects of very weak interactions made detectable by high energies, we limit ouselves primarily to a study of the the CPT-even interactions.2 (Both our examples are of this class.) In $\S 2.2$ we construct the most general CPT-even interaction for the standard model and show that it preserves anomaly cancellation. In $\S 2.3$ we discuss certain approximations that we will use later on. As an illuminating exercise, in $\S 2.4$ we work out the kinematics of $n$-body decays in the special case in which off-diagonal matrix elements of the velocity-mixing matrices may be neglected. Novel phenomena arise. For example, a decay can be kinematically allowed both at low and high energy, but forbidden for an intermediate range of energies.

The last section applies our formalism to various possibly observable manifestations of Lorentz violation. We discuss phenomena involving charged leptons in $§ 3.1$, in particular the possible appearance of radiative muon decay at high energies. We discuss phenomena involving neutrinos in $§ 3.2$, where we show how searches for neutrino oscillations at high energy and long baseline can provide new and powerful tests of special relativity. We discuss hadronic manifestations of Lorentz-violation in $\S 3.3$, especially those relating to ultra-high energy cosmic rays. An Appendix explains why, contrary to our earlier assertion [4], the observed absence of a velocity difference between right- and left-handed photons (which would violate $\mathrm{CPT}$ ) does not constrain the appearance of CPT-violating effects elsewhere.

\section{Generalities}

Here we develop the formalism needed to determine the observable consequences of Lorentz violation so as to obtain precise high-energy tests of special relativity.

\subsection{Building Lagrangians}

We first construct all CPT-even Lorentz-violating rotationally-invariant perturbations for a general renormalizable theory of scalars, spinors and gauge mesons. We also show

2 This argument would be evaded if the CPT-even couplings were on the order of the squares of the CPT-odd ones, expressed as dimensionless ratios to an appropriate mass scale. This would be consistent with both renormalization-group flow and our energy-growth rule, and would lead to the dominance of the CPT-odd interactions at moderately high energies. We mainly ignore this possibility here. 
that the matrix elements of these interactions grow with energy more rapidly than those of the CPT-odd Lorentz-violating interactions.

We begin by summarizing some well-known properties of the Lorentz group $S O(3,1)$ [6]. We assemble all the fields in the theory into a big vector $\Phi$. The action of $\Lambda$, an element of $O(3,1)$, on these fields is effected by a unitary operator in Hilbert space $U(\Lambda)$ :

$$
U(\Lambda)^{\dagger} \Phi(x) U(\Lambda)=D(\Lambda) \Phi\left(\Lambda^{-1} x\right)
$$

where $D(\Lambda)$ is some finite-dimensional representation of the Lorentz group.

The Lie algebra of $S O(3,1)$ may be written as the (complex) sum of two commuting angular momenta, $\vec{J}^{(+)}$and $\vec{J}^{(-)}$. An irreducible finite-dimensional representation of the group may be labeled by two half integers, $j_{+}$and $j_{-}$, and is of dimension $\left(2 j_{+}+1\right)\left(2 j_{-}+\right.$ 1). We sometimes write the fields forming the basis for the irreducible representation as $\Phi_{m_{+}, m_{-}}$, where $m_{ \pm}$is the eigenvalue of $J_{z}^{( \pm)}$.

The values of $\left(j_{+}, j_{-}\right)$are $(0,0)$ for a scalar; $(0,1 / 2)$ or $(1 / 2,0)$ for a Weyl spinor, depending on its chirality; $(1 / 2,1 / 2)$ for a 4 -vector; $(1,1)$ for a traceless symmetric tensor; and the direct sum of $(1,0)$ and $(0,1)$ for an antisymmetric tensor. The complex conjugates of a set of fields transforming according to $\left(j_{+}, j_{-}\right)$transform according to $\left(j_{-}, j_{+}\right)$.

For $R(\vec{e} \theta)$, a rotation about an axis $\vec{e}$ by an angle $\theta$,

$$
D(R(\vec{e} \theta))=\exp \left[-i\left(\vec{J}^{(+)}+\vec{J}^{(-)}\right) \cdot \vec{e} \theta\right]
$$

For $B(\vec{e} \phi)$, a Lorentz boost in a direction $\vec{e}$ by rapidity $\phi$,

$$
D(B(\vec{e} \phi))=\exp \left[\left(\vec{J}^{(+)}-\vec{J}^{(-)}\right) \cdot \vec{e} \phi\right]
$$

Finally, for the anti-unitary CPT operator $\Omega$,

$$
\Omega^{-1} \Phi(x) \Omega=(-1)^{2 j+} \Phi^{\dagger}(-x)
$$

We are ready to begin our analysis. From Eq. (2.2) we note that every rotationally invariant term $\mathcal{L}^{\prime}$ in the Lagrangian must lie in a representation for which $j_{+}=j_{-} \equiv j$. Elementary angular-momentum theory tells us that the term is:

$$
\mathcal{L}^{\prime} \propto \sum_{m=-j}^{j}(-1)^{m} \Phi_{m,-m} .
$$


If the state $|\psi\rangle$ is boosted in the $z$-direction by rapidity $\phi$, the expectation value of $\mathcal{L}^{\prime}$ is transformed according to:

$$
\left\langle\psi\left|U^{\dagger}\left(B\left(\vec{e}_{z} \phi\right)\right) \mathcal{L}^{\prime}(0) U\left(B\left(\vec{e}_{z} \phi\right)\right)\right| \psi\right\rangle \propto e^{2 j \phi}\left\langle\psi\left|\Phi_{j,-j}(0)\right| \psi\right\rangle+O\left(e^{(2 j-2) \phi}\right) .
$$

That is, $\left\langle\mathcal{L}^{\prime}\right\rangle$ grows at large energy like $E^{2 j}$. As we shall show shortly, the largest value of $j$ attainable with operators of renormalizable type is $j=1$, a traceless symmetric tensor, which is CPT-even. The only other rotationally-invariant possibilities are $j=1 / 2$, a vector, which is CPT-odd, and $j=0$, a Lorentz invariant scalar.

Let us begin by considering only scalar fields. With no loss of generality we can consider these to be all real. To attain $j=1$ we need at least two derivative operators, and for renormalizability we can have no more than two (and no more than two scalar fields). Thus the only possibility is

$$
\frac{1}{2} \sum_{a, b} \partial_{i} \phi^{a} \epsilon_{a b} \partial^{i} \phi^{b}
$$

where $\epsilon_{a b}$ is a real symmetric matrix and the sum runs over the scalar fields. (Of course, we could just as well have said that the only possibility is $\frac{1}{2} \sum_{a, b} \partial_{0} \phi^{a} \epsilon_{a b} \partial^{0} \phi^{b}$; the difference is a Lorentz invariant.)

Now let us consider spinors. With no loss of generality we may take our fundamental fields to be a set of $n(1 / 2,0)$ Weyl spinors, $u^{a}$; the conjugate fields , $u^{a \dagger}$, are then $(0,1 / 2)$ spinors. The only Lorentz-invariant interactions either couple two spinors of the same type or couple a spinor, a conjugate spinor, and a derivative. The most general free Lagrangian is

$$
u^{\dagger} i\left(\partial_{0}-\vec{\sigma} \cdot \vec{\partial}\right) Z u+\frac{1}{2} u^{T} \sigma_{y} M u+\frac{1}{2} u^{\dagger} \sigma_{y} M^{\dagger} u^{*},
$$

where $Z$ is a Hermitian $n \times n$ matrix and $M$ is a (possibly complex) symmetric matrix. We can always make a linear transformation such that $Z$ is one and $M$ is real, positive, and diagonal. We then have the theory of $n$ free spin- $1 / 2$ particles, each of which is its own antiparticle.

The only way to construct a renormalizable interaction with $j=1$ is to couple together a spinor, a conjugate spinor, and a derivative. Thus we obtain

$$
\frac{1}{2} \sum_{a, b} i \epsilon_{a b} u^{a \dagger} \vec{\sigma} \cdot \vec{\partial} u^{b}
$$

where $\epsilon_{a b}$ is a Hermitian matrix. 
A particularly simple case is that of two $(1 / 2,0)$ Weyl spinors carrying opposite charges under a $U(1)$ internal symmetry group. Then one Weyl spinor and the conjugate of the other can be joined to make a single Dirac bispinor, $\psi$, the most general free Lagrangian is the standard Dirac Lagrangian, and the most general $j=1$ interaction is

$$
\frac{1}{4} \bar{\psi} i \vec{\partial} \cdot \vec{\gamma}\left[\epsilon_{+}\left(1+\gamma_{5}\right)+\epsilon_{-}\left(1-\gamma_{5}\right)\right] \psi
$$

where $\epsilon_{ \pm}$are real numbers.

We now turn to gauge mesons. The couplings of the gauge mesons to the scalars and spinors is completely determined by gauge invariance, so we need only look at the gaugefield self-couplings. Let us begin with a single Abelian gauge field, and let us express it in terms of an electric and a magnetic field, defined, as usual, by $E^{i}=F^{0 i}$ and $B^{i}=\frac{1}{2} \epsilon^{i j k} F_{j k}$. Out of these we can make three independent rotational invariants of renormalizable type, 3 which we may choose to be $\vec{E}^{2}-\vec{B}^{2}, \vec{E} \cdot \vec{B}$, and $\vec{B}^{2}$. The first two are Lorentz invariants; we are left with $\vec{B}^{2}$, just as in the first example of $\S 1$.

A general gauge group is locally the product of simple factors and Abelian factors. Gauge invariance forbids cross terms between gauge fields belonging to different simple factors, so for each simple factor we have one interaction, of the form $\sum_{a} \vec{B}^{a} \cdot \vec{B}^{a}$, where the sum runs over the generators of the factor. For the Abelian factors we can have cross terms, but we can eliminate them by an orthogonal transformation on the Abelian generators; thus we again obtain one $B^{2}$ term for each factor.

\subsection{The Almost Lorentz-Invariant Standard Model}

As an example, let us construct the $j=1$ interactions in the standard model with three generations of quarks and leptons. There are only a few terms in the bosonic sector of the model: one $\vec{B}^{2}$ term for each factor of the gauge group and one velocity-mixing term for the Higgs doublet, for a total of four real parameters.

The number of parameters is much greater in the fermionic sector of the model. Each family of spinor fields transforms like the direct sum of five inequivalent irreducible representations of the $S U(3) \otimes S U(2) \otimes U(1)$ gauge group. Gauge invariance excludes cross terms between different irreducible representations, but not those between families. Thus we have five $3 \times 3$ Hermitian velocity-mixing matrices of the form (2.9), for 45 additional real parameters.

3 The CPT-odd term $\vec{A} \cdot \vec{B}$, where $\vec{A}$ is the vector potential, is discussed in the Appendix. 
A small reduction in the number of parameters may be effected by field redefinitions. We can rescale the space coordinates while leaving the time coordinate unchanged, thereby eliminating one of the gauge-field $\vec{B}^{2}$ terms. Finally, we note that the minimal standard model conserves each lepton flavor. Thus, we may multiply the lepton fields by a phase factor depending only on flavor so as to make two of the off-diagonal elements in one of the lepton velocity-mixing matrices real while not affecting the Lorentz-invariant Lagrangian. Thus the standard model involves a total of $4+45-3=46$ independent Lorentz-violating (but CPT-conserving) parameters. (We remark in passing that there are a comparable number of parameters corresponding to $j=1 / 2$ CPT-violating departures from Lorentz invariance.)

We used gauge invariance throughout this construction, but it has been the gauge invariance of the classical Lagrangian. Is this theory gauge-invariant as a quantum theory? Phrased another way, we know that anomalies cancel in the standard model, but does the cancellation persist when we take account of the 46 noninvariant terms? We demonstrate below that it does.

Our demonstration depends on the classic analysis of anomalies in Lorentz-invariant non-Abelian gauge theories [7]. This begins by showing that the full theory is anomalyfree (to all orders in perturbation theory) if the corresponding theory of massless spinors coupled to external c-number gauge fields is anomaly-free. If we assemble all the spinor fields into a column vector $u$, the Lagrangian for such a theory is:

$$
i u^{\dagger}\left(D_{0}-\vec{\sigma} \cdot \vec{D}\right) u
$$

where the covariant derivative $D_{\mu}$ is defined by $D_{\mu}=\partial_{\mu}+A_{\mu}$, with $A_{\mu}$ a matrix-valued field composed of the gauge fields with their associated coupling matrices. If we define a gauge transformation of the fields in the usual way,

$$
\delta u=\delta \omega u, \quad \delta A_{\mu}=\left[\delta \omega, A_{\mu}\right]-\partial_{\mu} \delta \omega
$$

where $\delta \omega$ is an infinitesimal gauge transformation, it may appear that the theory defined by (2.11) is gauge-invariant. However, this is not necessarily so. A carefully regulated computation of $W(A)$, the generating functional of connected Green's functions, yields

$$
\delta W=-\frac{1}{32 \pi^{2}} \operatorname{Tr} \int d^{4} x \epsilon^{\mu \nu \lambda \sigma} \delta \omega F_{\mu \nu} F_{\lambda \sigma}
$$

4 We were disappointed to discover this; we had hoped that the condition of anomaly cancellation would put some constraints on our 46 free parameters. 
where $F_{\mu \nu}=\partial_{\mu} A_{\nu}-\partial_{\nu} A_{\mu}+\left[A_{\mu}, A_{\nu}\right]$. Only if this expression ("the anomaly") vanishes is the theory in fact gauge-invariant. Projection operators on irreducible representations of the gauge group commute with $\delta \omega$ and $F_{\mu \nu}$; thus a convenient way to evaluate the trace is to sum the contributions of the irreducible multiplets. For the standard model, this sum vanishes.

We wish to extend all this to a theory with a Lorentz-violating interaction of the form (2.9). That is to say, (2.11) is replaced by

$$
i u^{\dagger}\left(D_{0}-\left(1-\frac{1}{2} \epsilon\right) \vec{\sigma} \cdot \vec{D}\right) u
$$

where $\epsilon$ is a Hermitian matrix acting only on the flavor indices. This replacement alters the high-momentum behavior of the spinor propagator and we must alter our regularization procedure accordingly. If we use massive regulator fields, the derivative terms in their Lagrangian must be of the form (2.14), not (2.11). Likewise, if we regulate the measure in the path integral, we must use the differential operator from (2.14), not from (2.11).

We must sum over irreducible multiplets, which may be chosen to be eigenspaces of $\epsilon$. In each eigenspace, the Lagrangian is of the form (2.14) with $\epsilon$ a number. We may introduce new variables (denoted by primes) by:

$$
\begin{aligned}
\vec{x} & =\left(1-\frac{1}{2} \epsilon\right) \vec{x}^{\prime}, & x_{0}=x_{0}^{\prime}, \\
\vec{A}^{\prime} & =\left(1-\frac{1}{2} \epsilon\right) \vec{A}, & A_{0}^{\prime}=A_{0} .
\end{aligned}
$$

In terms of these variables, the $\epsilon$ term disappears from the Lagrangian (and from the regularization procedure). The contribution of each multiplet to the anomaly is the same as it would be if $\epsilon$ were zero, except that unprimed variables are replaced by primed ones. This replacement has no effect on (2.13), which is invariant under general coordinate transformations. Thus the contributions of the irreducible multiplets are independent of $\epsilon$. If they cancel when $\epsilon$ vanishes (as they do), they cancel for general $\epsilon$.

\subsection{From Lagrangians to Particles}

Particle properties of free fields follow trivially from the Lagrangian, but things are more difficult for interacting fields. For simplicity we study the energy-momentum relation for one real scalar field of (renormalized) mass $m$. The generalization to more complicated systems is straightforward. If the theory is Lorentz-invariant, the inverse renormalized propagator has the form:

$$
-i D^{-1}=\left(p^{2}-m^{2}\right) A\left(p^{2}\right)
$$


for some function $A$. We normalize the field conventionally so that $A\left(m^{2}\right)=1$, then add a Lorentz-violating interaction to the theory with some small coefficient $\epsilon$, as in Eq. (2.7). We begin in the linear approximation, retaining terms only first order in $\epsilon$. Later we investigate whether this approximation is justified. The addition to $D^{-1}$ must transform like the 00 component of a traceless symmetric tensor. The only possibility is a multiple of $4 p^{0} p^{0}-g^{00} p^{2}=4 \vec{p}^{2}+3 p^{2}$. The $p^{2}$ term can be absorbed in $A$, whence (2.16) becomes:

$$
-i D^{-1}=\left(p^{2}-m^{2}\right) A\left(p^{2}\right)+\epsilon \vec{p}^{2} B\left(p^{2}\right),
$$

for some function $B$. It is convenient to normalize the Lorentz-violating interaction such that $B\left(m^{2}\right)=1$.

To first order in $\epsilon$, the shift in the zeroes of $D^{-1}$ is:

$$
p^{2}=E^{2}-\vec{p}^{2}=m^{2}+\epsilon \vec{p}^{2} .
$$

The energy-momentum relation may be rewritten in the seemingly conventional form $E^{2}=$ $\vec{p}^{2} c^{2}+m^{2} c^{4}$, with $c$ the maximal attainable velocity and $m c^{2}$ the rest energy of the particle. However, it must be remembered that $c^{2}$ has been changed by the factor to $1+\epsilon$ and $m^{2}$ has been diminished by a factor of $(1+\epsilon)^{2}$. Of course, the tiny mass shift is of no experimental interest, but this is very much not the case for the shift in $c^{2}$, as we saw in $\S 1$. Abrupt effects turn on when the dimensionless parameter $\epsilon \vec{p}^{2} / m^{2}$ is of order unity, while for gradual effects (like $\mu^{+} \rightarrow e^{+}+\gamma$ ) the energy at which the effect becomes significant can be many orders of magnitude smaller.

Even for gradual effects, $E$ is typically very large and we must ask whether new Lorentz-invariant physics at high energies might affect our predictions. Eq. (2.18) shows that this does not happen. Even though $E$ is large, $p^{2}$ remains $O\left(m^{2}\right)$ and the possible new physics remains irrelevant.

When can the linear approximation be trusted? It can certainly be trusted for free field theory, where it is exact. It can also be trusted for leptons and electroweak gauge mesons. For these particles, all couplings are weak, all radiative corrections are small, and all propagators are well approximated by those of free field theory.

Things are trickier for hadrons. A detailed investigation of QCD with Lorentznoninvariant terms is beyond our ken, but we can make an educated guess on the basis of a simple model. Let's take QCD and rescale the space coordinates but not the time coordinate, as in Eq. (2.15). In the new coordinates, we seem to have a noninvariant theory, but 
it's just QCD wearing a false beard. All that happens is that $p^{2}$ in Eq. (2.16) is replaced by $p^{2}-\epsilon \vec{p}^{2}$. Thus an expansion in powers of $\epsilon$ is in fact an expansion in powers of $\epsilon \vec{p}^{2} / \Lambda^{2}$, where $\Lambda$ is the QCD mass scale. Since typical hadron masses are $O(\Lambda)$, this implies that for gradual effects the linear approximation is a very good one, while for abrupt effects it is only a rough approximation. Of course, a rough approximation is not a useless one; it can give us a qualitative picture of what is going on, and even (with a modest amount of luck) yield correct order-of-magnitude quantitative predictions.

\subsection{The Kinematics of Particle Decays}

In this section we analyze the decay of a particle into $n$ other particles in our Lorentz non-invariant theories [8]. We make three simplifying assumptions: (1) that all particles are spinless. The extension to spin- $\frac{1}{2}$ particles is straightforward and has no effect on our conclusions; (2) that the linear approximation is valid; and (3) that the matrix elements of the invariance-violating perturbation between particles with different masses are negligible. Thus we obtain a set of particles each of which obeys an energy-momentum relation of the form (2.18). That is to say, the $a$ th particle has, in addition to its own mass, $m_{a}$, its own maximum attainable velocity ("its own velocity of light") $c_{a}$, and obeys the energymomentum relation:

$$
E_{a}^{2}=\vec{p}_{a}^{2} c_{a}^{2}+m_{a}^{2} c_{a}^{4}
$$

In what follows we use $a=0$ for the decaying particle and $a=1 \ldots n$ for the decay products.

A decay is kinematically permitted if we can arrange the decay products such that their total momentum is $\vec{p}_{0}$ and their total energy is $E_{0}$. Let $E_{\min }\left(\vec{p}_{0}\right)$ denote the minimum total energy of the decay products for given total momentum $\vec{p}_{0}$. The decay is possible if and only if:

$$
E_{0} \geq E_{\min }\left(\vec{p}_{0}\right)
$$

because if $E_{\min }<E_{0}$ we can obtain equality by adding opposite transverse components to two of the decay momenta.

If we delete the transverse components of all decay momenta, we lower the final-state energy without changing the total momentum. It follows that all momenta are collinear in the configuration of minimum total energy. We use this fact to simplify our analysis and replace $\vec{p}_{a}$ by $p_{a}$, the longitudinal (and only nonzero) component of $\vec{p}_{a}$. 
$E_{\text {min }}$ must be stationary under variations of the decay momenta that leave their sum unchanged. Introducing a Lagrange multiplier $u$, we must make

$$
\sum E_{a}-u\left(\sum p_{a}-p_{0}\right)
$$

stationary, where here and in the remainder of this section the sum is over the decay products. Differentiating with respect to $p_{a}$, we find:

$$
u=\frac{d E_{a}}{d p_{a}}=v_{a},
$$

where $v_{a}$ is the velocity of the ath particle. (We've used Hamilton's equations at the last step.) Thus all the decay particles move with a common velocity $u$. Furthermore. the relation:

$$
d E_{\min }=u \sum d p_{a}=u d p_{0}
$$

shows that $u=d E_{\min } / d p_{0}$.

We can now explore the limits of small and large $p_{0}$. For $p_{0}=0$, the minimum energy configuration is one in which all the decay momenta vanish; the decay is allowed if and only if:

$$
m_{0} c_{0}^{2} \geq \sum m_{a} c_{a}^{2}
$$

Of course, physics is certainly nearly Lorentz invariant, so that the $c_{a}$ 's can differ only very slightly. For all practical purposes, we can drop them from Eq. (2.24).

For very large $p_{0}, u$ must be ultrarelativistic and we can approximate $E_{a}$ by $c_{a} p_{a}$. The energy is minimized by giving all the momentum to the particle with the smallest $c$. In this limit, the decay is allowed if:

$$
c_{0}>\min _{a \neq 0} c_{a}
$$

and is forbidden if $c_{0}<\min c_{a}$. (We must go beyond the ultrarelativistic approximation to settle the question if $c_{0}=\min c_{a}$.)

Eqs. (2.24) and (2.25) are independent. A decay is allowed or forbidden in the high energy limit regardless of whether it is allowed or forbidden at low energies. It's interesting to ask what can happen at intermediate energies. For example, can there be alternating bands of allowed and forbidden energies? 
We begin by answering this question when all the decay products have nonzero masses; afterwards we'll extend our analysis to deal with massless particles. Let's rewrite the condition for allowed decay, $E_{0} \geq E_{\min }$, as:

$$
m_{0}^{2} c_{0}^{4} \geq E_{\min }^{2}\left(p_{0}\right)-c_{0}^{2} p_{0}^{2} \equiv Y\left(p_{0}\right)
$$

We shall prove that every stationary point of $Y$ is a local maximum. Differentiating (2.26) yields:

$$
\frac{1}{2} \frac{d Y}{d p_{0}}=E_{\min } u-c_{0}^{2} p_{0}=\sum\left(c_{a}^{2}-c_{0}^{2}\right) p_{a}
$$

where we have used $E_{a}=c_{a}^{2} p_{a} / u$ at the last step. The derivative vanishes at a stationary point, so at least one term in the sum must be negative, $c_{0}>\min _{a \neq 0} c_{a}$. Note that this condition coincides with Eq. (2.25), the condition that the process be allowed at high energy.

Differentiating once more, we find:

$$
\frac{1}{2} \frac{d^{2} Y}{d p_{0}^{2}}=\frac{d u}{d p_{0}} \sum\left(c_{a}^{2}-c_{0}^{2}\right) \frac{d p_{a}}{d u}=\frac{d u}{d p_{0}} \sum\left(c_{a}^{2}-c_{0}^{2}\right) \frac{p_{a} c_{a}^{2}}{u\left(c_{a}^{2}-u^{2}\right)}
$$

At a stationary point, we can use the vanishing of (2.27) to write this as:

$$
\begin{aligned}
\frac{1}{2} \frac{d^{2} Y}{d p_{0}^{2}} & =\frac{d u}{d p_{0}} \sum\left(c_{a}^{2}-c_{0}^{2}\right) \frac{p_{a}}{u}\left[\frac{c_{a}^{2}}{c_{a}^{2}-u^{2}}-\frac{c_{0}^{2}}{c_{0}^{2}-u^{2}}\right] \\
& =-u \frac{d u}{d p_{0}} \sum \frac{p_{a}\left(c_{a}^{2}-c_{0}^{2}\right)^{2}}{\left(c_{a}^{2}-u^{2}\right)\left(c_{0}^{2}-u^{2}\right)} .
\end{aligned}
$$

As $p_{0}$ increases monotonically from zero to infinity, $u$ increases monotonically from zero to $\min c_{a}$. Thus $d u / d p_{0}$ is positive, as are the factors $\left(c_{a}^{2}-u^{2}\right)$ and $\left(c_{0}^{2}-u^{2}\right)$, these last by (2.25). This completes the proof.

Because every stationary point of $Y$ is a local maximum, $Y$ can have at most one stationary point. If $Y$ has no stationary points, it is a monotone function of $p_{0}$ and can cross $m_{0}^{2} c_{0}^{4}$ at most once. This leads to four possibilities: (1) The decay is allowed at all energies. (2) The decay is forbidden at all energies. (3) The decay is allowed for all energies below a certain energy and forbidden for greater energies. (4) The decay is forbidden for all energies below a certain energy and allowed for all greater energies. If $Y$ has one stationary point, 目 it may cross $m_{0}^{2} c_{0}^{4}$ twice, the first time from below and the second time from above.

5 The necessary and sufficient conditions for $Y$ to have an extremum are Eq. (2.25) and $\sum\left(c_{a}^{2}-\right.$ $\left.c_{0}^{2}\right) m_{a}>0$ (which ensures that $Y\left(p_{0}\right)$ increases near $p_{0}=0$ ). 
This adds one more possibility: (5) The decay is forbidden for a certain band of energy but allowed for all energies above or below this band. There are no other possibilities.

Our arguments break down if there are massless particles among the decay products. (For example, we can no longer write energy and momentum as functions of velocity.) Nevertheless, we now show that our conclusions remain valid.

If there is more than one massless particle among the decay products, we can lower $E_{\text {min }}$ by giving all the momentum carried by the massless particles to the one with the smallest value of $c$. Thus no generality is lost by restricting ourselves to the case in which there is only one massless particle, which we label by $a=1$.

Consider a configuration with $p_{1}$ fixed and the remaining momentum, $p_{0}-p_{1}$, distributed among the massive particles so as to minimize their total energy. The total energy of this configuration is:

$$
E=c_{1} p_{1}+E_{\min }^{\prime}\left(p_{0}-p_{1}\right)
$$

where $E_{\min }^{\prime}$ is the minimum energy computed for the massive particles only. Thus,

$$
d E / d p_{1}=c_{1}-u^{\prime}\left(p_{0}-p_{1}\right)
$$

where $u^{\prime}$, the common velocity of the massive particles, is a monotone increasing function of their total momentum. There are two cases: (1) $u^{\prime}\left(p_{0}\right)<c_{1}$, so that $E$ is a monotone increasing function of $p_{1}$ and $E_{\min }=E_{\text {min }}^{\prime}\left(p_{0}\right)$. The massless particle carries no momentum so that the analysis is the same as in the massive case. $(2) u^{\prime}\left(p_{0}\right)>c_{1}$, which is possible only if:

$$
c_{1}<\min _{a \neq 1} c_{a}
$$

for otherwise $u^{\prime}$ cannot reach $c_{1}$. In this case, increasing $p_{1}$ lowers $E$ until $u^{\prime}$ reaches $c_{1}$, and

$$
E_{\min }=c_{1}\left(p_{0}-p_{0}^{\prime}\right)+E_{\min }^{\prime}\left(p_{0}^{\prime}\right)
$$

where $p_{0}^{\prime}$ is defined by $u^{\prime}\left(p_{0}^{\prime}\right)=c_{1}$. This analysis proceeds as in the massive case up to and including Eqs. (2.27) and (2.25), but the computation of $d^{2} Y / d p_{0}^{2}$ is different. Because only $p_{1}$ depends on $p_{0}$,

$$
\frac{1}{2} \frac{d^{2} Y}{d p_{0}^{2}}=\left(c_{1}^{2}-c_{0}^{2}\right) \frac{d p_{1}}{d p_{0}}=\left(c_{1}^{2}-c_{0}^{2}\right) c_{1}<0
$$


by Eqs. 2.25 and (2.32). Just as in the case of only massive decay products, every stationary point of $Y$ is a local maximum. Thus we reach the same conclusions here as we did there.

\section{Applications}

Here we examine effects of CPT-conserving departures from Lorentz invariance on the behavior of ultra-relativistic particles. Our treatment of leptonic phenomena (in $\S 3.1$ and $\S 3.2)$ is not subject to the qualifications discussed in $\S 1.4$. However, the hadronic phenomena (in $\S 3.3$ ) often involve hadron energies so large that $E \sqrt{\delta c^{2}}$ is comparable to the QCD mass scale. Thus, our treatment of the $c_{a}$ as energy-independent parameters is not always justified. This error affects the values of the Lorentz-violating parameters needed to produce novel phenomena, but not the phenomena themselves.

\subsection{Phenomena Involving Charged Leptons}

Tests of Lorentz Invariance from Photon Stability: Suppose that the velocity of light exceeds the maximal attainable velocity (MAV) of electrons, i.e., $c_{\gamma}>c_{e}$, where for the moment we ignore possible flavor and helicity dependences. It follows [4] that photons of sufficient energy are unstable. In particular, the decay:

$$
\gamma \rightarrow e^{-}+e^{+}
$$

becomes kinematically permitted when the photon energy $E$ exceeds $2 m_{e} / \sqrt{\delta_{\gamma e}}$, where $\delta_{\gamma e} \equiv c_{\gamma}^{2}-c_{e}^{2}$. The decay rate of photons well above this threshold is $\Gamma_{e e} \simeq \frac{1}{2} \alpha \delta_{\gamma e} E$. The fact that primary cosmic-ray photons with energies up to $20 \mathrm{TeV}$ have been detected lets us set the limit $c_{\gamma}-c_{e}<10^{-15}$. Note that $\left|\delta_{\gamma e}\right|$ is not so well constrained: the absence of vacuum Čerenkov radiation by electrons with energies up to $500 \mathrm{GeV}$ sets the limit: $c_{e}-c_{\gamma}<5 \times 10^{-13}$. In a similar manner, much weaker constraints may be placed on the MAVs of the heavier charged leptons.

6 The borderline situation, where the stationary point of $Y$ occurs at $p_{0}=p_{0}^{\prime}$, must be treated separately. In this case we must use the analysis for $p_{0}<p_{0}^{\prime}$ to compute $d^{2} Y / d p_{0}^{2}$ from below and that for $p_{0}>p_{0}^{\prime}$ to compute it from above. The two answers don't agree because the second derivative of $Y$ is not continuous at the stationary point, but it doesn't matter because both are negative. 
More stringent tests of Lorentz invariance might be imagined to result from the stability of photons under decay into two neutrinos, for which the threshold energy depends on tiny neutrino masses. This mode is forbidden in the standard model, which attributes neither masses nor magnetic moments to neutrinos, but there is a considerable body of empirical evidence for neutrino oscillations, and hence for neutrino masses. The non-standard physics responsible for neutrino masses could generate neutrino magnetic moments via loop diagrams, thereby enabling the decays $\gamma \rightarrow \nu+\nu^{\prime}$ and $\bar{\nu}+\bar{\nu}^{\prime}$ (with $\nu$ and $\nu^{\prime}$ necessarily distinct chiral neutrinos).

The decay rate of a photon with energy $E$ well above threshold into neutrinos is: $\Gamma_{\nu \nu^{\prime}} \sim \lambda^{2} \mu_{B}^{2} \delta_{\gamma \nu}^{2} E^{3}$, where the flavor-changing "magnetic moment" of the neutrino is $\lambda \mu_{B}$ with $\mu_{B} \equiv e / 2 m_{e}$ (the Bohr magneton). We assume $c_{\nu}=c_{\nu^{\prime}}$ and put $\delta_{\gamma \nu}=c_{\gamma}^{2}-c_{\nu}^{2}$. Because the magnetic interaction is dimension-five (rather than dimension-four, like the electric), $\Gamma_{\nu \nu^{\prime}}$ is quadratic in $\delta_{\gamma \nu}$ rather than linear.7 It follows that the range of an energetic photon is cosmological even if the neutrino magnetic moment is (implausibly) set equal to its experimental upper limit [9]: $\lambda=2 \times 10^{-10}$. Consequently, no strong bound on $c_{\gamma}-c_{\nu}$ can be deduced from observations of energetic cosmic-ray photons.

Radiative Muon Decay? The decay mode mode $\mu \rightarrow e+\gamma$ is often searched for but never found. This is not surprising: it is forbidden in the minimal standard model. Although induced by radiative corrections in models with neutrino masses, the expected branching ratio is far too small to be detected. However, and as we noted elsewhere [10] [11], Lorentz-violating perturbations that are not flavor diagonal can lift the accidental symmetry ordinarily preventing radiative decay.

Recall that the velocity eigenstates of high-energy leptons do not in general coincide with their mass eigenstates at low energy. In the following analysis, we ignore possible mixings of electrons and muons with tau leptons. The relevant portion of the Lagrangian takes the following form in the preferred frame:

$$
\left(\begin{array}{ll}
\bar{\mu} & \bar{e}
\end{array}\right) \vec{\gamma} \cdot(\vec{p}-e \vec{A})\left\{\mathcal{C}_{R} \frac{1}{2}\left(1+\gamma_{5}\right)+\mathcal{C}_{L} \frac{1}{2}\left(1-\gamma_{5}\right)\right\}\left(\begin{array}{c}
\mu \\
e
\end{array}\right)
$$

7 Our estimates of $\Gamma_{e e}$ and $\Gamma_{\nu \nu^{\prime}}$ are lowest order in the appropriate Lorentz-invariant operators, with photons satisfying the Lorentz-violating dispersion relation $E^{2}-\vec{p}^{2}=\delta E^{2} \equiv$ " $m_{\gamma}^{2}$ ". The decay rates are $\left.\Gamma_{e e}\right|_{\text {rest }} \sim \alpha m_{\gamma}$ and $\left.\Gamma_{\nu \nu^{\prime}}\right|_{\text {rest }} \sim\left(\lambda \mu_{B}\right)^{2} m_{\gamma}^{3}$ in the "photon rest frame." These expressions yield our results when boosted to the lab frame. 
where $\mu$ and $e$ denote fields corresponding to mass eigenstates. The matrices $\mathcal{C}_{L, R}$ (which would be unit matrices were Lorentz symmetry unbroken) are:

$$
\mathcal{C}_{a} \equiv \frac{1}{2}\left(\begin{array}{cc}
2 \bar{c}_{a}+\delta c_{a} \cos 2 \theta_{a} & \delta c_{a} \sin 2 \theta_{a} \\
\delta c_{a} \sin 2 \theta_{a} & 2 \bar{c}_{a}-\delta c_{a} \cos 2 \theta_{a}
\end{array}\right), \quad a=L, R
$$

The mixing angle $\theta_{L}$ determines the velocity eigenstates of left-handed leptons (or righthanded antileptons) whose MAVs are $\bar{c}_{L} \pm \frac{1}{2} \delta c_{L}$. Similarly, $\theta_{R}$ determines the velocity eigenstates of right-handed leptons (or left-handed antileptons), whose MAVs differ by $\delta c_{R}$. All four maximal velocities are known to be very close to $c_{\gamma}$, the velocity of light in vacuum.

Electroweak gauge invariance implies that the matrix $\mathcal{C}_{L}$ appears in the kinetic-energy of neutrinos as well as charged leptons. In $\S 3.2$ we show how the parameters $\bar{c}_{L}, \delta c_{L}$ and $\theta_{L}$ may be constrained by experiments or observations involving neutrinos.

For the analysis to follow, it is convenient to define the small parameters:

$$
\epsilon_{a}^{2} \equiv\left|\delta c_{a} \sin 2 \theta_{a}\right|^{2}, \quad a=L, R
$$

The flavor-changing terms in Eq. (3.2), namely

$$
\frac{1}{2}\left(\epsilon_{R}+\epsilon_{R}\right) \bar{\mu} \vec{\gamma} \cdot(\vec{p}-e \vec{A}) e+\frac{1}{2}\left(\epsilon_{R}-\epsilon_{R}\right) \bar{\mu} \vec{\gamma} \cdot(\vec{p}-e \vec{A}) \gamma_{5} e+\text { h.c. }
$$

induce the decay process $\mu \rightarrow e+\gamma$. We shall see that its helicity-dependent partial decay rate is $\sim \alpha \epsilon_{a}^{2} M \gamma^{3}$, where $M$ is the muon mass and $\gamma$ is its Lorentz factor in the preferred frame. This rate increases with the cube of the muon energy rather than falling with $1 / E$. Thus flavor-changing Lorentz-violations, if they are present, will cause $\mu \rightarrow e+\gamma$ to become the dominant decay mode of muons at sufficiently high energies.

The rate of radiative muon decay is controlled by the muon energy and the magnitudes of the Lorentz-violating parameters. Otherwise, it is a first-order electromagnetic effect, not a weak decay. Its branching ratio is $B \sim \alpha \epsilon_{a}^{2} \gamma^{4} M \tau_{0}$, where $\tau_{0}$ is the lifetime of a muon at rest. Departures from Lorentz invariance also modify the rates of allowed processes, such as $\pi \rightarrow \mu+\nu$ and $\mu \rightarrow e+\nu+\bar{\nu}$, but in these cases the conventional decay rates $\Gamma_{0} / \gamma$ and their Lorentz-violating corrections (of the form $\epsilon^{2} \gamma^{3} \Gamma_{0}$ ) are both intrinsically weak. They do not involve the enormous enhancement factor $\alpha M \tau_{0} \simeq 2.6 \times 10^{15}$ that appears in the branching ratio for radiative muon decay. That's why the most sensitive tests of Lorentz invariance in this context are obtained from the study of muons, and in particular, from the search for a lifetime anomaly of muons at ultra-high energies. 
The interaction (3.5), treated in lowest order perturbation theory, yields the rate for radiative muon decay. A straightforward but tedious computation (generously carried out for us by Mark Wise) yields the following result for its branching ratio when the muon is at rest:

$$
B=\frac{\alpha M \tau_{0}}{4}\left(\epsilon_{L}^{2}+\epsilon_{R}^{2}\right) \simeq 6.4 \times 10^{14}\left(\epsilon_{L}^{2}+\epsilon_{R}^{2}\right) .
$$

The current experimental limit [12], $B<4.9 \times 10^{-11}$, yields an upper limit on the relevant Lorentz-violating parameter:

$$
\epsilon_{L}^{2}+\epsilon_{R}^{2}<8 \times 10^{-26} \quad \text { from muon decay at rest. }
$$

The branching ratio for Lorentz-violating radiative muon decay is a rapidly increasing function of the energy. Direct searches for it do not seem feasible. Nonetheless one might detect the onset of this mode through its effect on the muon lifetime, which for ultrarelativistic left-handed $\mu^{-}$(or right-handed $\mu^{+}$) is:

$$
\tau_{L}(\gamma)=\frac{\gamma \tau_{0}}{1+b_{L} \gamma^{4}}, \quad \text { where } \quad b_{L} \equiv \frac{\alpha M \tau_{0}}{30}\left(68 \epsilon_{R}^{2}+\epsilon_{L}^{2}\right)
$$

and for ultrarelativistic right-handed $\mu^{-}$(or left-handed $\mu^{+}$) is:

$$
\tau_{R}(\gamma)=\frac{\gamma \tau_{0}}{1+b_{R} \gamma^{4}}, \quad \text { where } \quad b_{R} \equiv \frac{\alpha M \tau_{0}}{30}\left(68 \epsilon_{L}^{2}+\epsilon_{R}^{2}\right)
$$

(These calculations were also carried out for us by Mark Wise.) At sufficiently high energy, the lifetime of muons with either helicity decreases with $\gamma^{-3}$ rather than increasing with $\gamma$.

The CERN $g-2$ experiment, aside from measuring the muon's anomalous magnetic moment, offers a precise test of the energy dependence of its lifetime. At $\gamma=29.3$ (corresponding to the 'magic energy' at which the experiment was performed) the results confirm the expected time-dilation of thed muon lifetime to an accuracy of one part in a thousand [13]. Because the muons in the ring favor neither helicity when time-averaged, we obtain the limit $b_{L}+b_{R}<2.7 \times 10^{-9}$, or:

$$
\epsilon_{L}^{2}+\epsilon_{R}^{2}<5 \times 10^{-25} \quad \text { from muon } g-2
$$

which is inferior to that obtained from the direct search, Eq. (3.7), but not by much! (The agreement between theoretical and experimental values of $g-2$ provides an independent but much weaker test of Lorentz invariance.) 
The Muon Collider-A Threat Averted! Lorentz-violating effects consistent with the above constraints could interfere with the operation of a future muon collider. In [10] and [11], we took as a necessary condition for its proper functioning, that the muon decay rate at the design energy of the collider must not exceed twice its expected value. This criterion, assuming unpolarized beams, translates to $\frac{1}{2}\left(b_{L}+b_{R}\right) \gamma^{4}<1$. Thus a $1 \mathrm{TeV}$ collider requires $b_{L}+b_{R}<2 \times 10^{-16}$ ( or $\epsilon_{L}^{2}+\epsilon_{R}^{2}<3 \times 10^{-32}$ ). The constraints discussed above are much weaker than this. However, we suggested that constraints sufficient to protect the muon collider might be obtained through studies of cosmic rays.

The highest energy cosmic-ray muons arise from forward decays of secondary pions. These are mostly longitudinally polarised $\mu_{R}^{-}$and $\mu_{L}^{+}$, so that searches for a lifetime anomaly of these muons can set a bound on $b_{R}$, which is proportional to the linear combination $68 \epsilon_{L}^{2}+\epsilon_{R}^{2}$. Although $\epsilon_{L}$ can be severely constrained by observations of neutrino oscillations, it's possible that $\epsilon_{R} \gg \epsilon_{L}$. Absent any a priori knowledge of the ratio $\epsilon_{L} / \epsilon_{R}$, a bound on $b_{R}$ yields a 69 -fold weaker bound on $b_{R}+b_{L}$

In particular, we suggested [10] how a comparison of underground muon fluxes with different flight times but the same slant depth in rock might yield the required constraint. Following our suggestion, V.S. Narasimham and M.R. Krishnaswamy [14] have analyzed data taken at the Kolar Gold fields. Their preliminary result is $b_{R}<8 \times 10^{-18}$. An even stronger constraint has been obtained by R. Cowsik and B.V. Sreekantan [15] from their considerations of data concerning horizontal air showers. They obtain the remarkably strong bound $b_{R}<10^{-25}$. From Eq. (3.9), this bound corresponds to:

$$
68 \epsilon_{L}^{2}+\epsilon_{R}^{2}<10^{-39} \quad \text { from air showers . }
$$

The results to either of these recent cosmic-ray analyses are sufficient to safeguard the muon collider from a weakening of the relativistic dilation of the muon lifetime. Indeed, taken by themselves, they provide sensitive tests of special relativity. For example, the air shower result (with $\epsilon_{L}=\epsilon_{R}$ and maximal velocity mixing of muons and electrons) yields the following constraint on the difference of maximal velocities of the two velocity eigenstates:

$$
\left|c^{\prime}-c\right|_{e \mu}<4 \times 10^{-21} .
$$

We shall show in $\S 3.2$ how neutrino physics can do even better. 


\subsection{Phenomena Involving Neutrinos}

Although differences among neutrino velocities are severely constrained directly (by studies of neutrino oscillations, discussed below) and indirectly (from constraints involving muons or electrons discussed in $\S 3.1$ via $S U(2)$ gauge invariance), there are only weak constraints on neutrino maximal velocities as such. The current limit, $\left|c_{\nu}-c_{\gamma}\right|<10^{-8}$, results from the detection of neutrinos from supernova 1987a [16]. A stronger limit, $\left|c_{\nu}-c_{\gamma}\right|<2 \times 10^{-16}$, may be set if neutrinos from gamma-ray bursts at cosmological distances could be detected [17], but this result pales in comparison to other tests of Lorentz invariance that have been (or could be) set.

Lorentz-Violating Neutrino Oscillations? We showed in [4] and [11] how CPTconserving Lorentz violations lead to neutrino oscillations even if neutrinos are massless. However, observable neutrino oscillations may result from a combination of effects involving neutrino masses and Lorentz violation.

Neutrinos with modest energy (even solar neutrinos) are nevertheless ultra-relativistic particles because neutrino masses are known to be small. For this reason, searches for neutrino oscillations can provide exquisitely sensitive tests of Lorentz invariance. We assume there exist three chiral neutrinos with Majorana masses given by the complex symmetric matrix $m$ in a flavor-diagonal basis. Conventional neutrino oscillations are described in terms of the Hermitian squared-mass matrix $m^{2}=m m^{\dagger}$. In particular, observable oscillation effects depend on two differences of squared masses and four parameters akin to the Kobayashi-Maskawa angles and phase in the quark sector.

With Lorentz symmetry violated, the description of neutrino oscillations becomes much more complicated. Although we usually neglect CPT-violating interactions, in this case it is hardly any work to include them. Thus, in addition to our usual velocity-mixing term, parameterized by c, a $3 \times 3$ Hermitian matrix of maximum attainable velocities, we allow the most general CPT-odd symmetry violating interaction, $u^{\dagger} b u$, where $\mathrm{b}$ is also a $3 \times 3$ Hermitian matrix. The energies of ultra-relativistic neutrinos with definite momentum $p$ are the eigenvalues of the matrix:

$$
c p+m^{2} / 2 p+b
$$

8 Similar effects can result from violations of the equivalence principle rather than special relativity [18. 
Neutrino energy eigenstates in the limit of high energy are the eigenvectors of the matrix $c$, just as at low energy they are the eigenvectors of the matrix $m^{2}$.

To avoid undue complexity, we limit ourselves to a discussion of two-flavor neutrino oscillations. Imagine neutrinos to be produced with a definite momentum and flavor $\left(\nu_{\ell}\right.$, where $\ell=e$ or $\mu$ ) and detected after travelling a distance $R$ through empty space. Their oscillations satisfy a seemingly conventional formula:

$$
P\left(\nu_{\ell} \rightarrow \nu_{\ell}\right)=1-\sin ^{2} 2 \Theta \sin ^{2}\{\Delta R / 4\}
$$

where the mixing angle $\Theta$ and phase factor $\Delta$ appearing in Eq. (3.14) (and expressed in the flavor basis) are given implicitly in terms of eight convention-independent parameters:

$$
\begin{aligned}
& \Delta \sin 2 \Theta=\delta m^{2} \sin 2 \theta_{m} / E+2 \delta b e^{i \eta} \sin 2 \theta_{b}+2 \delta c e^{i \eta^{\prime}} E \sin 2 \theta_{c} \\
& \Delta \cos 2 \Theta=\delta m^{2} \cos 2 \theta_{m} / E+2 \delta b \cos 2 \theta_{b}+2 \delta c E \cos 2 \theta_{c} .
\end{aligned}
$$

The parameters characterizing the oscillations are: three mixing angles, $\theta_{m}, \theta_{b}$ and $\theta_{c}$, two complex phases, $\eta$ and $\eta^{\prime}$, and the differences between the eigenvalues of the matrices $m^{2}$, $b$, and $c$, denoted respectively by $\delta m^{2}, \delta b$ and $\delta c$. (Note that $\delta c$ here is the same as $\delta c_{L}$ in §3.1.) To illustrate the possibilities inherent in Eqs. (3.14) and (3.15), we mention a few special cases of Lorentz-violating two-flavor neutrino oscillations:

$$
\begin{aligned}
P\left(\nu_{\ell} \rightarrow \nu_{\ell}\right) & =1-\frac{\sin ^{2}\left\{\left(\delta m^{2} R / 4 E\right) \sqrt{1+\left(E / E_{0}\right)^{2}}\right\}}{1+\left(E / E_{0}\right)^{4}} \\
& =1-\frac{\sin ^{2}\left\{(\delta v R E / 2) \sqrt{1+\left(E_{0} / E\right)^{2}}\right\}}{1+\left(E_{0} / E\right)^{4}} \\
& =1-\sin ^{2} 2 \theta \sin ^{2}\left\{R\left(\delta m^{2} / 4 E+\delta b / 2+\delta c E / 2\right)\right\}
\end{aligned}
$$

where $E_{0} \equiv \delta m^{2} /(2 \delta c)$. Eq. (3.16a) corresponds to $\theta_{m}=\pi / 4$ with $\delta b=\sin 2 \theta_{c}=0$. It yields maximal mass oscillations for $E \ll E_{0}$, but essentially none for $E \gg E_{0}$. Eq. (3.16b) corresponds to a converse case with $\theta_{c}=\pi / 4$ and $\delta b=\sin 2 \theta_{m}=0$ : maximal velocity oscillations at high energy, but none at low energy.

To obtain Eq. (3.16d), we set all three mixing angles equal and put $\eta=\eta^{\prime}=0$. In this case, the energy-momentum eigenstates are independent of energy. This example encompasses all three scenarios discussed by Foot, Leung and Yasuda [19] for atmospheric neutrino oscillations - each of which, they say, is consistent with the atmospheric neutrino data reported by Super-Kamiokande [20]. 
Conventional neutrino oscillations depend only on $R / E$, the ratio of the flight-length of the neutrino to its energy. If Lorentz symmetry is violated, the dependence on these parameters is more complicated. Nonetheless, neutrino experiments performed at a variety of energies can severely constrain the Lorentz-violating parameters. Let's give a simple example related to accelerator searches for $\nu_{\mu}-\nu_{e}$ oscillations. The strongest current limit on $\delta m^{2}$ (with $\sin ^{2} 2 \theta_{m} \sim 1$ ), $\delta m^{2}<0.09 \mathrm{eV}^{2}$, follows from a relatively low energy experiment [21]. Higher energy neutrino experiments, such as [22], offer less stringent constraints on $\delta m^{2}$ but are better suited to search for Lorentz-violating velocity oscillations. From that experiment, and assuming $\sin ^{2} \theta_{m} \sim 1$, we find for the difference of the maximal velocities of the two velocity eigenstates:

$$
\left|c^{\prime}-c\right|_{\nu_{e} \nu_{\mu}}<6 \times 10^{-22}
$$

Finally, we note that stringent constraints on the CPT-violating parameters that affect neutrino oscillations have been obtained from altogether different laboratory experiments. According to one of its collaborators [23], the spectroscopic test of Lorentz invariance described in [3] constrains the parameter $b_{3}$ (as defined by Colladay and Kostalecký [5]). They obtain $\left|b_{3}\right|<7 \times 10^{-19} \mathrm{eV}$ for electrons and $\left|b_{3}\right|<1.2 \times 10^{-21} \mathrm{eV}$ for nucleons. The

former result, expressed in our model and in the preferred frame, corresponds loosely 9 to the constraint $\left|b_{e e}\right|<3 \times 10^{-16} \mathrm{eV}$. This result suggests that CPT-violating effects are too small to affect neutrino oscillations, except when the source distance far exceeds the diameter of the Earth (as in the case of solar or extra-solar neutrinos).

\subsection{Phenomena Involving Hadrons}

To each particle species we assign a maximal attainable velocity $c_{a}$. That is, we assume that a dispersion relation of the form $E^{2}=c_{a}^{2} p^{2}+m_{a}^{2} c_{a}^{4}$ describes a particle of type $a$ moving freely in the preferred frame. Many Lorentz-violating (but CPT-conserving) phenomena may be described in terms of the purely kinematic effects of these parameters. For simplicity, we ignore the helicity dependence of the MAVs, although it could easily be taken into account. Flavor-changing effects are not relevant to the phenomena discussed in this section and are likewise ignored.

The Neutral Kaon System: Lorentz-violating effects can be abrupt or gradual. We gave examples of each in $\S 1$ - the sudden onset of vacuum Cerenkov radiation by energetic

9 Here we neglect possible CPT violations involving right-handed electrons. 
protons, and an energy-dependent modulation of the behavior of neutral kaons. Although our primary focus here is on abrupt hadronic effects, it is illustrative to examine the latter phenomenon in more detail. We consider the special case in which the velocity and mass eigenstates of neutral kaons coincide, and in which the MAVs of $K_{L}$ and $K_{S}$ are not the same: $c_{K_{L}}-c_{K_{S}} \neq 0$. This leads to an energy dependence of their apparent mass difference, as determined by observations of time-dependent interference phenomena:

$$
\Delta M=\left.\Delta M\right|_{\text {rest }}+M \beta \gamma^{2}\left(c_{K_{L}}-c_{K_{S}}\right),
$$

where $\gamma$ and $\beta$ are the Lorentz factor and velocity of the decaying kaons. A careful analysis of the experimental data carried out by Hambye, Mann and Sarkar [24] yields the bound:10

$$
\left|c_{K_{L}}-c_{K_{S}}\right|<3 \times 10^{-21}
$$

Stable Neutral Pions? Let's turn to abrupt phenomena. A simple example involves neutral pions and photons. Suppose that $c_{\gamma}-c_{\pi^{0}}>0$. The process $\pi^{0} \rightarrow 2 \gamma$ (the dominant decay mode of neutral pions) becomes kinematically forbidden for pions with energies exceeding $E=m_{\pi} / \sqrt{c_{\gamma}^{2}-c_{\pi}^{2}}$. Conversely, photons with energies significantly above $E$ will decay rapidly according to the scheme: $\gamma \rightarrow \gamma+\pi^{0}$. This example is not entirely academic. Suppose, for example:

$$
c_{\gamma}-c_{\pi^{0}}=c_{\gamma}-c_{e}=c_{\gamma}-c_{\pi^{0}}=10^{-22} .
$$

For this case, all modes of $\pi^{0}$ decay are kinematically forbidden at pion energies exceeding $10^{19} \mathrm{eV}$. Thus ultra-high-energy (UHE) primary cosmic rays may include neutral pions (if they are stabilized by tiny departures from Lorentz invariance), but not photons (which would be destabilized by the same mechanism).

Stable Neutrons? Ordinarily, neutron decay $\left(n \rightarrow p+e^{-}+\bar{\nu}\right)$ is allowed but proton beta decay $\left(p \rightarrow e^{+}+n+\nu\right)$ is kinematically forbidden. As we have seen in $\S 2.4$, departures from Lorentz invariance can affect the kinematics of decay processes. They even can invert this pattern! To see how this can come about, we examine the case $c_{p}=c_{e}=c_{\nu}<c_{n}$.

10 Other tests of CPT and Lorentz invariance using neutral mesons are discussed in [24] and elsewhere 25]. 
Conventional relativistic kinematics may be used in this example (with $c_{p}$ as "the speed of light"), provided that the neutron is assigned an effective mass $m_{\text {eff }}$ given by:

$$
m_{\mathrm{eff}}^{2} \equiv m_{n}^{2}-\left(c_{p}^{2}-c_{n}^{2}\right) \vec{p}^{2},
$$

where $\vec{p}$ is its momentum in the preferred frame. Neutron beta-decay is allowed if and only if $m_{\mathrm{eff}}>m_{p}+m_{e}$. Expressed in terms of the neutron energy $E$ in the preferred frame, this condition becomes:

$$
E<E_{1}=\sqrt{\frac{m_{n}^{2}-\left(m_{p}+m_{e}\right)^{2}}{c_{p}^{2}-c_{n}^{2}}} \simeq 2.7 \times 10^{19}\left[\frac{10^{-24}}{c_{p}-c_{n}}\right]^{1 / 2} \mathrm{eV} .
$$

With our choice of Lorentz-violating parameters, neutrons with energies exceeding $E_{1}$ are stable particles that can be present among UHE cosmic rays.

In a similar manner, we can deduce the necessary and sufficient condition for proton beta decay to be kinematically permitted. It is:

$$
E>E_{2} \simeq \sqrt{\frac{m_{n}^{2}-\left(m_{p}-m_{e}\right)^{2}}{c_{p}^{2}-c_{n}^{2}}} \simeq 4.1 \times 10^{19}\left[\frac{10^{-24}}{c_{p}-c_{n}}\right]^{1 / 2} \mathrm{eV},
$$

with $E$ the proton energy in the preferred frame. For this partcular example, protons with energies exceeding $E_{2}$ are unstable particles that cannot be present among UHE cosmic rays.

The above results are expressed in terms of a nominal choice, $c_{p}-c_{n}=10^{-24}$, lying beyond the sensitivity of current tests of Lorentz invariance. Thus it is conceivable that the highest energy cosmic-ray primaries are stable neutrons.

Evading the GZK Cutoff? Soon after the discovery of the cosmic background radiation (CBR), Greisen [26] and Zatsepin and Kuz'min [27] saw how it limits the propagation of UHE cosmic rays. Primary nucleons with sufficient energy will suffer inelastic impacts with CBR photons. This results in what is known as the GZK cutoff, saying that nucleons with energies $>5 \times 10^{19} \mathrm{eV}$ cannot reach us from further than $\sim 50 \mathrm{Mpc}$. However, the primary cosmic-ray energy spectrum seems to extend well beyond $10^{20} \mathrm{eV}$ [28].

The mechanism producing UHE cosmic rays is unknown. Exotic origins have been suggested, such as: topological defects, active galactic nuclei, and gamma-ray bursts [29]. These schemes are constrained or ruled out by the GZK cutoff. Other explanations are designed to evade the GZK cutoff: a primary flux of magnetic monopoles [30], "Z-boson 
bursts" produced by collisions of cosmic UHE neutrinos off relatively nearby relic neutrinos [31], and decay products of hypothetical super-heavy relic particles [32].

We have little to say about the origin of UHE cosmic rays. Rather, we point out that there may not be a GZK cutoff after all. Tiny departures from Lorentz invariance, too small to have been detected otherwise, have effects that increase rapidly with energy and can kinematically prevent cosmic-ray nucleons from undergoing inelastic collisions with CBR photons. The cutoff thereby undone, a deeply cosmological origin of UHE cosmic rays becomes tenable 33 .

To see how the GZK cutoff is affected by Lorentz violation, consider the formation reaction yielding the first pion-nucleon resonance:

$$
p+\gamma(\mathrm{CBR}) \longrightarrow \Delta(1232)
$$

by which a nucleon with energy $E$ collides inelastically with a CBR photon of energy $\omega$. The target photon energies are a thermal distribution with temperature $T=2.73 \mathrm{~K}$, or $k T \equiv \omega_{0}=2.35 \times 10^{-4} \mathrm{eV}$. For a head-on impact, the condition Eq. (2.20) determining whether the reaction can take place is approximately:

$$
2 \omega+\frac{M_{p}^{2}}{2 E} \geq\left(c_{\Delta}-c_{p}\right) E+\frac{M_{\Delta}^{2}}{2 E}
$$

where $c_{\Delta}-c_{p}$ is the relevant Lorentz-violating parameter. If $c_{\Delta}=c_{p}$, Eq. (3.25) yields the usual threshold energy for this process, $E_{f}=\left(M_{\Delta}^{2}-M_{p}^{2}\right) / 4 \omega$. Otherwise it yields a quadratic inequality in $E$ which can be satisfied if and only if $\left(c_{\Delta}-c_{p}\right)<\hat{\delta}(\omega) \equiv \omega / 2 E_{f}$. As $c_{\Delta}-c_{p}$ is increased toward $\hat{\delta}$, the threshold for the formation reaction grows toward $2 E_{f}$. However, if it exceeds its critical value,

$$
c_{\Delta}-c_{p}>\frac{2 \omega^{2}}{M_{\Delta}^{2}-M_{p}^{2}} \simeq 1.7 \times 10^{-25}\left[\omega / \omega_{0}\right]^{2},
$$

reaction (3.24) becomes kinematically forbidden for all $E$. Recalling that the photon spectrum is thermal, we see that if $c_{\Delta}-c_{p} \sim \hat{\delta}\left(\omega_{0}\right)$, the GZK cutoff due to resonant $\Delta(1232)$ formation would be relaxed. Should it much exceed this value, the formation reaction would be precluded off virtually all CBR photons.

Reaction (3.24) is the dominant process leading to the GZK cutoff as originally formulated. However, if $\Delta(1232)$ formation is not possible, a weakened version of the cutoff may result from non-resonant photo-production:

$$
p+\gamma(\mathrm{CBR}) \longrightarrow p+\pi
$$


If $c_{\pi}=c_{p}$, the threshold energy for pion production is $E_{p}=M_{\pi}\left(2 M_{p}+M_{\pi}\right) / 4 \omega$. However, as $c_{\pi}-c_{p}$ is increased from zero, the threshold grows. As $E \rightarrow \infty$, the pion energy $E_{\pi}$ must remain finite. Eq. (2.20) yields the kinematic condition for reaction (3.27) to occur:

$$
2 \omega \geq\left(c_{\pi}-c_{p}\right) E_{\pi}+\frac{m_{\pi}^{2}}{2 E_{\pi}} .
$$

This condition may be satisfied if and only if:

$$
c_{\pi}-c_{p}<\tilde{\delta}(\omega) \equiv \frac{2 \omega^{2}}{m_{\pi}^{2}} \simeq 5 \times 10^{-24}\left[\omega / \omega_{0}\right]^{2} .
$$

Reaction (3.27), and multiple pion production as well, are kinematically forbidden at all proton energies if $c_{\pi}-c_{p}>\tilde{\delta}(\omega)$. For the actual case of a thermal gas of photons, $c_{\pi}-c_{p} \sim \tilde{\delta}\left(\omega_{0}\right)$ would suppress photo-pion production, or even eliminate it entirely so that no vestige of the cutoff survives. 1 .

At present, lacking detailed observations of the highest energy cosmic rays and more precise tests of special relativity, we must regard as an intriguingly open question whether there is a GZK cutoff, and consequently, whether cosmic rays with energies above the cutoff can travel cosmological distances.

Finally we note that tiny departures from Lorentz invariance, such as we have discussed earlier in this Section, can explain the remarkable correlation discovered by Farrar and Biermann: that the five highest energy cosmic ray events appear to point toward compact radio-loud quasars [34]. We suggest that these events could have been produced by UHE primary neutrons arising from sources at large redshift. These particles could be stable if $c_{p}>c_{n}$; they could be immunized against the GZK cutoff if $c_{\pi}>c_{n}$. They are undeflected by intergalactic magnetic fields because they are neutral.

\section{Conclusions}

A wide variety of experiments and observations offer very precise tests of special relativity. Many of these results can be interpreted in terms of differences of MAVs of different particles, such as would result from Lorentz-violating (but CPT-conserving) perturbations

11 Note that much larger (and experimentally intolerable) violations of Lorentz invariance would be needed to produce a noticeable effect on the interactions of UHE cosmic rays with nuclei in the atmosphere. 
of the standard-model Lagrangian. The strongest constraints of this kind of which we are aware have been mentioned earlier and are listed below:

$$
\begin{aligned}
c_{p}-c_{\gamma} & <1 \times 10^{-23} & \S 1 & \text { [1] } \\
\left|c_{m}-c_{\gamma}\right| & <6 \times 10^{-22} & \S 1 & \text { 2] } \\
\left|c^{\prime}-c\right|_{\nu_{e} \nu_{\mu}} & <6 \times 10^{-22} & \S 3.2 & {[22} \\
\left|c^{\prime}-c\right|_{\mu e} & <4 \times 10^{-21} & \S 3.1 & \S 3.3
\end{aligned}
$$

Two of these constraints result from cosmic-ray observations, the others from experiments performed at very high (accelerator) energies, or in one case, at low energy. They are consistent with strict Lorentz invariance to a precision of more than twenty-one decimal places. Possible symmetry violations, if present at all, must be exceedingly small. How much further must experimenters test special relativity; when is enough enough? Our analysis addresses this question.

We have seen that maximal velocity differences lying two or three orders of magnitude below the current bounds can produce dramatic observable effects. Some would suppress or forbid the processes underlying the GZK cutoff, thereby permitting UHE cosmic-ray nucleons to travel cosmological distances. Another could stabilize UHE cosmic-ray neutrons, which would point toward their astrophysical sources. Maximal velocity differences of neutrinos can help to explain the observed properties of both solar and atmospheric neutrinos.

Fortunately, much can be done. Further observations of UHE cosmic rays are essential. They may confirm a predicted 'bump' just below the cutoff [35] resulting from products of inelastic collisions of primary protons with CBR photons, thereby providing evidence for the GZK cutoff. Or, they could belie the cutoff by confirming the Farrar-Biermann contention [34] that the highest energy events originate at cosmological distances. Dedicated searches for velocity oscillations of solar neutrinos, or of accelerator-produced $\sim \mathrm{TeV}$ neutrinos at baselines of $\sim 1000 \mathrm{~km}$, could reveal Lorentz-violating neutrino velocity differences as small as $10^{-25}$. Finally, laboratory searches for diurnal anisotropies more sensitive than any done before have become feasible [36].

\section{Acknowledgements}


We thank Mark Wise for his assistance with several computations, R. Cowsik and B.V. Sreekantan for graciously conveying their results (as well as those of V.S. Narasimham and M.R. Krishnaswamy) to us prior to publication, Larry Hunter for alerting us to the precision experiment in which he was a collaborator, and Barry Barish, George Field, Giorgio Giacomelli, Roman Jackiw, Alan Kosteleck'y, and Jean Zinn-Justin for enlightening discussions. This work was supported in part by the National Science Foundation under grant NSF-PHYS-92-18167.

\section{Appendix. Wrong Reasoning Made Right}

In our first paper on this subject [4] we advanced an argument for neglecting CPT-odd Lorentz-violating interactions.

The argument was based on studies [37] of an electromagnetic interaction proportional to $\frac{1}{2} \epsilon_{0 i j k} A^{i} F^{j k}=\vec{A} \cdot \vec{B}$. (This term is not gauge-invariant but it makes a gauge-invariant contribution to the action.) The new term makes an addition to the space-space part of the photon self-energy:

$$
\Pi_{i j}(p) \rightarrow \Pi_{i j}(p)+i \mu \epsilon_{i j k} p^{k}
$$

where $\mu$ is a constant with dimensions of mass. The added term causes vacuum birefringence; the experimental absence of this effect in radio-astronomy observations of distant quasars and radio galaxies leads to a very stringent upper bound, $\mu \leq 10^{-33} \mathrm{eV}$. In [4] we argued that any CPT-odd Lorentz-violating interaction would induce an addition to the Lagrangian proportional to $\vec{A} \cdot \vec{B}$. Thus the extreme smallness of $\mu$ is evidence for the extreme smallness of all CPT-odd interactions, and further searches for CPT-violating effects are pointless. Unfortunately, this argument is invalid.

Let us consider interactions for which the Lagrange density (not just the action) is invariant under electromagnetic gauge transformations; for brevity, we'll refer to these simply as "gauge-invariant interactions". We'll show that to first order in any gaugeinvariant CPT-odd interaction, and to any order in the Lorentz-invariant interactions, $\mu$ vanishes. (This confirms the expectation that gauge-invariant terms in the Lagrange density cannot induce gauge-noninvariant ones.) Thus the fact that $\mu$ is known to be tiny offers no strong constraint on the magnitude of gauge-invariant CPT-violating interactions.

It's useful to analyze a simple example before giving our proof in full generality. Let $\Gamma_{\mu \nu}(p, q)$ be the one-particle irreducible (1PI) Green's function for two photons and 
one Lorentz-violating interaction Lagrangian. Here the photon with index $\mu(\nu)$ carries momentum $p(q)$. (Thus the Lorentz-violating interaction carries momentum $-(p+q)$.) In first order in the Lorentz-violating interaction

$$
\Pi_{\mu \nu}(p)=\Gamma_{\mu \nu}(p,-p)
$$

Because the Lorentz-violating interaction is assumed to be gauge invariant, $\Gamma$ obeys the Ward identities

$$
p^{\mu} \Gamma_{\mu \nu}(p, q)=0
$$

and

$$
q^{\nu} \Gamma_{\mu \nu}(p, q)=0
$$

Now let us assume we compute $\Gamma$ by summing only those diagrams that have no internal photons. These diagrams have only massive-particle 12 internal lines; thus, in this approximation, $\Gamma$ is analytic at $p=q=0$. Differentiating (A.3a) with respect to $p^{\mu}$ and setting $p=0$, we find $\Gamma_{\mu \nu}(0, q)=0$. Thus every nonvanishing term in the Taylor expansion of $\Gamma$ has at least one factor of $p$; $\Gamma$ is $O(p)$. The same reasoning applied to (A.3b) tells us that $\Gamma$ is $O(q)$. Since $p$ and $q$ are independent variables, $\Gamma$ is $O(p q)$. Thus, by (A.2), the addition to the self-energy is $O\left(p^{2}\right)$ and makes a vanishing contribution to $\mu$.

The proof we have given rested on analyticity at vanishing momentum. Unfortunately, internal photons can produce singularities at precisely this point. In the remainder of this appendix we study these singularities and prove the following:

Theorem: Let $\Gamma_{\mu_{1} \ldots \mu_{n}}^{(n)}\left(p_{1} \ldots p_{n}\right)$ denote the $n$-photon 1PI Green's function, and let us introduce a uniform scaling parameter $\lambda$ by replacing $p_{a}$ by $\lambda p_{a}$. Then to either zeroth or first order in the CPT-odd interaction, and to any finite order in the Lorentz-invariant interactions, and for any positive $\epsilon, \Gamma^{(n)}$ vanishes as $\lambda$ goes to zero more rapidly than $\lambda^{n-\epsilon}$. (Note that our announced result, $\mu=0$, is a corollary of this theorem for $n=2$.)

The proof proceeds by induction in the number of internal photon lines.

We begin with the case of no internal photon lines. In this case $\Gamma^{(n)}$ is analytic at vanishing external momentum, and, to first order in the Lorentz-violating interactions, the argument is a straightforward generalization of that for the simple example above. We treat the $n$ external momenta as independent variables, letting the Lorentz-violating

12 Neutrino masses may be tiny on the scale of high-energy physics, but they're enormous on the scale of radio waves. 
interaction carry off the momentum inserted at the photon lines. We then use the Ward identities to show that the leading term in the Taylor series is $O\left(p_{1} \ldots p_{n}\right)=O\left(\lambda^{n}\right)$.

This doesn't work in zeroth order in Lorentz violation, because there is no Lorentzviolating interaction to carry off the momentum. The best we can do is to choose $q_{1} \ldots q_{n-1}$ as our independent variables and use the Ward identities to show that the leading term is $O\left(p_{1} \ldots p_{n-1}\right)=O\left(\lambda^{n-1}\right)$. However, the term zeroth order in Lorentz violation is Lorentz invariant, and there is no Lorentz-invariant way to construct a rank $n$ tensor as a multilinear function of $n-1$ independent vectors. Thus this term must vanish, and the leading term is at best $O\left(\lambda^{n}\right)$.

To prove the inductive step, we need some information from the once well-known theory of Feynman-diagram singularities [38]. For real external momenta, the case of interest here, the theory can be reduced to a set of simple algorithms: (1) A given Feynman graph generates a family of reduced graphs, each obtained by taking some proper subset of the internal lines of the graph and contracting them to points. (2) The reduced graph leads to a singularity if it can be interpreted as a diagram of a classical process occuring in space-time, with all particles (that is to say, all uncontracted internal lines) on the mass shell and moving forward in time. (3) If there is a singularity, the associated discontinuity is calculated by the usual Feynman rules except that $\left(p^{2}-m^{2}+i \epsilon\right)^{-1}$ is replaced by $\delta\left(p^{2}-m^{2}\right) \theta\left(p^{0}\right)$ in the propagators for the uncontracted internal lines.

We are now ready to use induction. We assume the theorem is true for $r$ or fewer internal photon lines and consider the singularities of graphs with $r+1$ internal photons. Since all energies inserted into the graph are arbitrarily small, there is not enough energy to create a massive particle and all massive-particle lines must be contracted. Thus the reduced graph contains only photon lines, joined together at vertices which represent contracted subgraphs of the original graph. If we sum, in some fixed order of perturbation theory, all graphs leading to the same reduced graph, the vertices become the $\Gamma$ 's at the appropriate order of pertubation theory. (We're being a bit sloppy here: subgraphs of a 1PI graph need not be 1PI, so the vertices can contain tree graphs. However, it's easy to check that these have no effect on the power-counting of the next paragraph.)

Because some of the internal photons in the original graph must be uncontracted, an $m$-line vertex in the reduced graph must have $r$ or fewer internal photon lines and, by the inductive hypothesis, must vanish at small $\lambda$ more rapidly than $\lambda^{m-\epsilon}$. It will be convenient to consider this as one factor of $\lambda^{1-\epsilon}$ for each photon line attached to the vertex. We can now compute the $\lambda$-dependence of the discontinuity. Every external photon contributes 
a factor of $\lambda^{1-\epsilon}$. Every internal photon contributes a factor of $\lambda^{2-\epsilon}$ from its two ends and a factor of $\lambda^{-2}$ from the $\delta$-function, yielding no net contribution. Every independent loop integration contributes a factor of $\lambda^{4}$. Thus the discontinuity vanishes faster than $\lambda^{n+4 L-\epsilon}$, where $\mathrm{L}$ is the number of loops. This in turn vanishes faster than $\lambda^{n-\epsilon}$, which is the result we need. (It is critical that we are computing the discontinuity and not the full $\Gamma$. The $\delta\left(p^{2}\right) \theta\left(p^{0}\right)$ propagators in the reduced graph keep the internal momenta small when the external momenta are small and legitimize the use of a small-momentum bound for the vertices.)

Once we have the discontinuity, we can construct a function with that discontinuity, for example, by integrating a dispersion relation. This function also vanishes faster than $\lambda^{n-\epsilon}$. Thus $\Gamma^{(n)}$ is the sum of a singular function that vanishes faster than $\lambda^{n-\epsilon}$ and a function that is free of singularities, that is to say, an analytic function. But we can use the same arguments for the analytic function that we used for the case of no internal photon lines to show that it vanishes like $\lambda^{n}$. This completes the proof. 


\section{References}

[1] E. g., M. P. Hagen and C. M. Will, Phys. Today 40 (May 1987), 69.

[2] S. K. Lamoreaux, J. P. Jacobs, B. R. Heckel, F. J. Raab, and E. N. Fortson, Phys. Rev. Lett. 57 (1986) 3125.

[3] C.J. Berglund, L.R. Hunter, D. Krause, Jr., E.O. Prigge, and M.S. Romfeldt, Phys. Rev. Lett. 75 (1995) 1879.

[4] S. Coleman and S. L. Glashow, Phys. Lett. B405 (1997) 249.

[5] D. Colladay and V.A. Kostelecký, Phys. Rev. D55 (1997) 6760, D58 (1998) 116002, and earlier references therein.

[6] E.g., S. Weinberg, The Quantum Theory of Fields I, $\S 4.6$ and $\S 4.8$, Cambridge U. Press, 1995.

[7] W. Bardeen, Phys. Rev. 184 (1969) 1848; S. Adler and W. Bardeen, Phys. Rev. 182 (1969) 1517; W. Bardeen and B. Zumino, Nucl. Phys. B244 (1984) 421.

[8] Versions of this section appear in: S.L. Glashow, in Proc. TAUP-97 Nucl.Phys. B70 Proc. Suppl. (1999) 70; S. L. Glashow, in Proc. First Tropical Workshop, San Juan, Puerto Rico, ed. José F. Nieves, AIP Conf. Proc. 444 (1998) 119.

[9] A.I. Derbin et al., Phys. At. Nucl. 57 (1994) 222.

[10] S. Coleman and S.L. Glashow, HUTP-98/A076

[11] S. Coleman and S.L. Glashow, HUTP-98/A080, to appear in Proc. Neutrino '98, Takayama, Japan.

[12] R.D. Bolton, Phys. Rev D38 (1988) 2077.

[13] E.g., F. Combley, F. J. M. Farley and E. Picasso, Phys. Rep. 68 (1981) 93.

[14] As reported to us by B.V. Sreekantan, private communication.

[15] R. Cowsik and B.V. Sreekantan, hep-ph/9811241 (to appear in Physics Letters B), and private communication.

[16] L. Stodolsky, Phys. Lett. B201 (1988) 353.

[17] E. Waxman and J. Bahcall, Phys. Rev. Lett. 78 (1997) 2292.

[18] M. Gasperini, Phys. Rev. D38 (1988) 2635;

A. Halprin and C.N. Leung, Phys. Rev. Lett. 67 (1991) 1833.

[19] R. Foot, C.N. Leung, and O. Yasuda, hep-ph/9809458.

[20] T. Kajita (for the SuperKamiokande Collaboration), to appear in Proc. Neutrino '98, Takayama, Japan.

[21] C. Angelini et al., Phys. Lett. B179 (1986) 307.

[22] E.B. Brucker et al., Phys. Rev. D34 (1986) 2183.

[23] L.H. Hunter, private communication.

[24] T. Hambye, R.B. Mann and U. Sarkar, Phys. Lett. B421 (1998) 105.

[25] V. A. Kostelecký, Phys. Rev. Lett. 80 (1998) 1818 and Indiana University preprint IUHET 396 hep-ph/9810352. 
[26] K. Greisen, Phys. Rev. Lett. 16 (1966) 748.

[27] G.T. Zatsepin and V.A. Kuz'min, JETP Lett. 41 (1966) 78.

[28] AGASA Collab. e.g., M. Takeda et.al., Phys. Rev. Lett. 81 (1998) 1163;

Fly's Eye Collab. e.g., D.J. Bird et al., Astrophys. J. 424 (1995 144;

Haverah Park Collab. See: M.A. Lawrence et al., J. Phys. G17 (1991) 733;

Yakutsk Collab. e.g., N.N. Efimov et al., in 22nd Intl. Cosmic Ray Conf. (1991) Dublin.

[29] See M. Takeda [28] and V. Berezinsky, Nucl. Phys. Proc. Suppl. 70 (1999) 419 for further references in these connections.

[30] T.W. Kephart and T.J. Weiler, Astro. Part. Phys. 4 (1996) 271.

[31] T.J. Weiler, hep-ph/9710431.

[32] V. Berezinsky, M. Kachelrieß, and V. Vilenkin, Phys. Rev. Lett. 79 (1997) 4302.

[33] A preliminary version of this section appears as S. Coleman and S.L. Glashow, HUTP98/A075, hep-ph/9808446.

[34] G.R. Farrar and P.L. Biermann, Phys. Rev. Lett. 81 (1998) 3579.

[35] C.T. Hill and D.N. Schramm, Phys. Rev. D31 (1985) 564.

[36] E.N. Fortson, private communication.

[37] S. Carroll, G. Field and R. Jackiw, Phys. Rev. D41 (1990) 1231;

M. Goldhaber and V. Trimble, J. Astrophys. Astr. 17 (1996) 17. See also: J.F.C. Wardle, R.A. Perley and M.H. Cohen, Phys. Rev. Lett. 79 (1997) 1801; J.P. Leahy, astro-ph/9704285; S. Carroll and G. Field, Phys. Rev. Lett. 79 (1997) 2394.

[38] R. Eden, P. Landshoff, D. Olive, and J. Polkinghorne, The Analytic S-Matrix, Cambridge University Press, 1966. 